\title{
Effect of activated charcoal hemoperfusion on renal function in patients with paraquat poisoning
}

\author{
LIANG SUN, PENG-BO YAN, YIN ZHANG, LU-QING WEI and GUO-QIANG LI \\ Department of Respiratory and Critical Care Medicine of Pingjing Hospital, Affiliated Hospital of \\ Logistics University of Chinese People's Armed Police Force, Tianjin 300162, P.R. China
}

Received October 19, 2016; Accepted June 6, 2017

DOI: $10.3892 /$ etm.2018.5712

\begin{abstract}
The present study retrospectively analyzed 19 patients diagnosed with paraquat (PQ) poisoning with the aim to investigate the effect of activated charcoal hemoperfusion on renal function and PQ elimination. The results indicated that 7 patients died and 12 survived. Non-oliguric renal failure occurred in all of the 7 patients who died. Among the 12 surviving patients, 10 had normal renal function and 2 developed non-oliguric renal failure. There was a linear correlation between plasma and urine paraquat concentration prior to and during activated charcoal hemoperfusion. The equation parameters together with the correlation coefficient on admission were as follows: $\mathrm{Y}=0.5820+1.7348 \mathrm{X}\left(\mathrm{R}^{2}=0.678\right.$; $\mathrm{F}=35.768 ; \mathrm{P}<0.0001)$. The equation parameters together with the correlation coefficient were as follows during activated charcoal hemoperfusion: $\mathrm{Y}=0.6827+1.2649 \mathrm{X}\left(\mathrm{R}^{2}=0.626\right.$; $\mathrm{F}=50.308 ; \mathrm{P}<0.0001)$. Therefore, it was concluded that in patients with normal renal function, the elimination kinetics of PQ by the kidneys were only associated with the plasma PQ concentration. Activated charcoal hemoperfusion had little effect on avoiding acute kidney injury in patients with severe PQ poisoning.
\end{abstract}

\section{Introduction}

Paraquat (PQ) is an effective and commercially important herbicide widely used throughout the world. However, the mortality rate of $\mathrm{PQ}$ poisoning has been reported to range from 50 to $90 \%$ when the pesticide is ingested either accidentally or intentionally as a suicide attempt (1). A previous study suggested that absorbed PQ requires removal from the

Correspondence to: Dr Guo-Qiang Li, Department of Respiratory and Critical Care Medicine of Pingjing Hospital, Affiliated Hospital of Logistics University of Chinese People's Armed Police Force, 220 Cheng-Lin Road, Tianjin 300162, P.R. China

E-mail: sunliangys@sina.com

Key words: paraquat, poisoning, kidney, metabolism, activated charcoal hemoperfusion bloodstream in order to improve the survival rate of patients with severe PQ poisoning (2).

PQ is not metabolized to any extent and is rapidly excreted in the urine after PQ poisoning even at low doses. The kidneys are effective at eliminating PQ but are vulnerable to PQ injury $(3,4)$. Therefore, elimination of $P Q$ simply relying on the kidney is slow, and removal of PQ from the blood by activated charcoal hemoperfusion (HP) in the first 12-15 h following ingestion may be beneficial (5). Various studies have indicated that HP is more efficient in the clearance of plasma PQ than the kidneys (2). Therefore, activated charcoal HP is widely used in the treatment of PQ poisoning in China (6).

Although the kidneys and HP are the two major routes of eliminating PQ following ingestion $(7,8)$, renal excretion is considered to be the major natural pathway of PQ elimination. Therefore, the initial renal function is an important factor for survival.

Application of HP has been reported to accelerate the removal of PQ from the blood. However, to the best of our knowledge, no previous study has focused on the effect of HP on renal function and PQ elimination by the kidneys. The present study determined the effect of HP on renal function and PQ elimination via the kidneys.

\section{Materials and methods}

Patients. A total of 19 patients with PQ poisoning were respectively observed in the present study. The subject selection criteria included the following: i) Oral PQ poisoning; ii) no acute kidney injury on admission; and iii) age of $<65$ years and $<18$ years. Potential participants were excluded if they were initially treated at a different hospital, or had any known cardiac, pulmonary or other chronic disease associated with a certain degree of renal failure as an underlying condition of $\mathrm{PQ}$ poisoning. Acute kidney injury was defined by a serum creatinine (SCr) level of $>97 \mu \mathrm{mol} / 1$ and blood urea nitrogen (BUN) levels of $>8.3 \mathrm{mmol} / \mathrm{l}$.

Treatment of the patients. All patients received HP therapy (Braun Diapact CRRT machine; Braun GmbH, Kronberg im Taunus, Germany) until plasma PQ levels became undectable, and then received continuous veno-venous hemofiltration therapy for $12 \mathrm{~h}$ after HP. To prevent absorption of PQ via the gastrointestinal tract, gastric lavage was performed via a 
nasogastric tube using $1 \mathrm{~g} / \mathrm{kg}$ activated charcoal in $500 \mathrm{ml} 0.9 \%$ saline for one time every $4 \mathrm{~h}$. Furthermore, Smecta (Beaufour Ipsen Pharmacy Co., Ltd., Tianjin, China) and magnesium sulfate (Huairen Pharmacy Co., Ltd., Tianjin, China) powder were placed into $20 \%$ mannitol (Shuanghe Pharmacy Co., Ltd., Tianjin, China) was administered via the anus.

Other treatments included intravenous infusion of cyclophosphamide (Guangdong Qingping Pharmacy Co., Ltd., Guangzhou, China), methylprednisolone sodium succinate injection (Pfizer, Inc., New York, NY, USA) and intravenous injections of dexamethasone (Jilin Extrawell Changbaishan Pharmaceutical Co., Ltd., Jilin, China). Vitamin E capsules (Xinyi Pharmaceutical Co., Ltd., Shanghai, China), metoprolol (Astra Zeneca, London, UK) and vitamin E injections (Zhongjing Biotechnology Co., Ltd., Harbin, China) were also administered.

Data collection. The following data were collected: Demographical factors (age, sex and medical history), initial BUN, $\mathrm{SCr}$, plasma and urine PQ concentration within $12 \mathrm{~h}$ of admission to the intensive care unit. Samples of plasma and urine were collected every $3 \mathrm{~h}$ while HP was performed. BUN and $\mathrm{SCr}$ were measured with a full automatic biochemical analyzer (AU5800; Beckman Coulter, Inc., Brea, CA, USA). Quantitative analysis of the plasma and urine PQ concentration was performed at the hospital laboratory by a gas chromatography method (9).

Statistical analysis. SPSS statistical software package 20.0 (IBM Corp., Armonk, NY, USA) and GraphPad Prism v4.0 (GraphPad Software, Inc., La Jolla, CA, USA) were used to perform statistical analysis. Values are expressed as the mean \pm standard deviation. Categorical variables are expressed as numbers or percentages for each item. Statistically significant differences between the two groups were analyzed using the independent two-samples t-test or the Mann-Whitney U test. The Chi-square test was used to assess the association between treatment protocols and survival rate. $\mathrm{P}<0.05$ was considered to indicate a statistically significant difference.

\section{Results}

Demographic and laboratory data. Demographic and laboratory results of the patients are summarized in Table I. A total of 19 patients were analyzed, including 8 females and 11 males (mean age, $40.37 \pm 12.55$ years; range, 25-63 years). A total of 7 patients died from pulmonary fibrosis and 12 survived. There were no significant inter-group differences in age or time between poisoning and HP therapy.

The renal function of all patients was normal on admission, and that of 16 patients remained normal after HP. However, non-oliguric renal failure occurred in all of the 7 patients who died. Among the 12 surviving patients, 10 had a normal renal function, while 2 (subjects nos. 5 and 16) developed non-oliguric renal failure (Table I).

The group of patients who did not survive had a higher initial plasma PQ concentration and longer time of activated charcoal HP therapy than the group of survivors. All patients exhibited a progressive increase in $\mathrm{SCr}$ and BUN levels during hospitalization. However, there were no significant inter-group differences in BUN at baseline, as well as $\mathrm{SCr}$ and BUN after HP. However, the group of non-survivors had a higher $\mathrm{SCr}$ and BUN than the survivors at discharge (Table II).

Effect of activated charcoal HP on the kidney toxicokinetics of paraquat. Plasma and urine PQ concentrations prior to and during HP are presented in Fig. 1. The plasma PQ concentration decreased rapidly when HP was performed. The concentrations of PQ in urine were associated with the plasma PQ concentration. When the plasma PQ concentration was $>0.5 \mathrm{mg} / \mathrm{l}$, the urine PQ concentration decreased in parallel with the plasma PQ concentration. Fig. 1 also revealed that urinary PQ concentrations were almost 2 times greater than plasma PQ concentrations when the plasma PQ concentration was $>0.5 \mathrm{mg} / \mathrm{l}(\mathrm{P}<0.05)$. When the plasma PQ concentration was $<0.5 \mathrm{mg} / \mathrm{l}$, the urine PQ concentration tended to be equal to the plasma PQ concentration.

The association between the plasma and urine PQ concentration prior to and during HP is presented in Fig. 2. At baseline, there was a linear association between plasma and urine PQ concentration. The equation parameters together with the correlation coefficient on admission were as follows: $\mathrm{Y}=0.5820+1.7348 \mathrm{X}\left(\mathrm{R}^{2}=0.678 ; \mathrm{F}=35.768 ; \mathrm{P}<0.0001\right)$. However, during HP, there was also a linear correlation between blood and urine PQ concentration. The equation parameters together with the correlation coefficient were as follows: $\mathrm{Y}=0.6827+1.2649 \mathrm{X}\left(\mathrm{R}^{2}=0.626 ; \mathrm{F}=50.308 ; \mathrm{P}<0.0001\right)$.

\section{Discussion}

In the present study, HP was demonstrated to effectively eliminate PQ. The kidney toxicokinetics of PQ during HP were assessed, which were previously not well assessed in humans. In the present study, urinary and plasma PQ concentrations were continuously monitored while HP was performed. A linear correlation was identified between the plasma and urine PQ concentration while HP was performed. When the plasma PQ concentration was $>0.5 \mathrm{mg} / \mathrm{l}$, urinary concentrations of $\mathrm{PQ}$ were nearly 2 times greater than the plasma concentrations. It was suggested that the kidney toxicokinetics of PQ at a given time may be interpreted along with the plasma levels of PQ, and are not associated with HP.

However, these results did not agree with those of Ikebuchi (10), who reported that urinary concentrations of PQ were 3.3-4.5 times greater than the plasma concentrations if renal function was normal. This discrepancy is likely due to differences in test methods and study populations. Another reason may be that $\mathrm{HP}$ eliminates $\mathrm{BUN}$ and $\mathrm{SCr}$, which affects the judgment of renal function.

Theoretically, accelerated removal of PQ from the blood should have a protective effect on the kidney. However, $\mathrm{SCr}$ and BUN of all patients displayed a progressive increase during hospitalization. Non-oliguric acute renal failure still occurred in 9 patients in spite of a rapid decrease in the PQ concentrations being observed. Not only the 7 patients who died, but also two of the surviving patients developed non-oliguric renal failure. However, no severe renal failure occurred in the survival group. These results suggested that HP has positive effects on avoiding kidney damage in patients with 


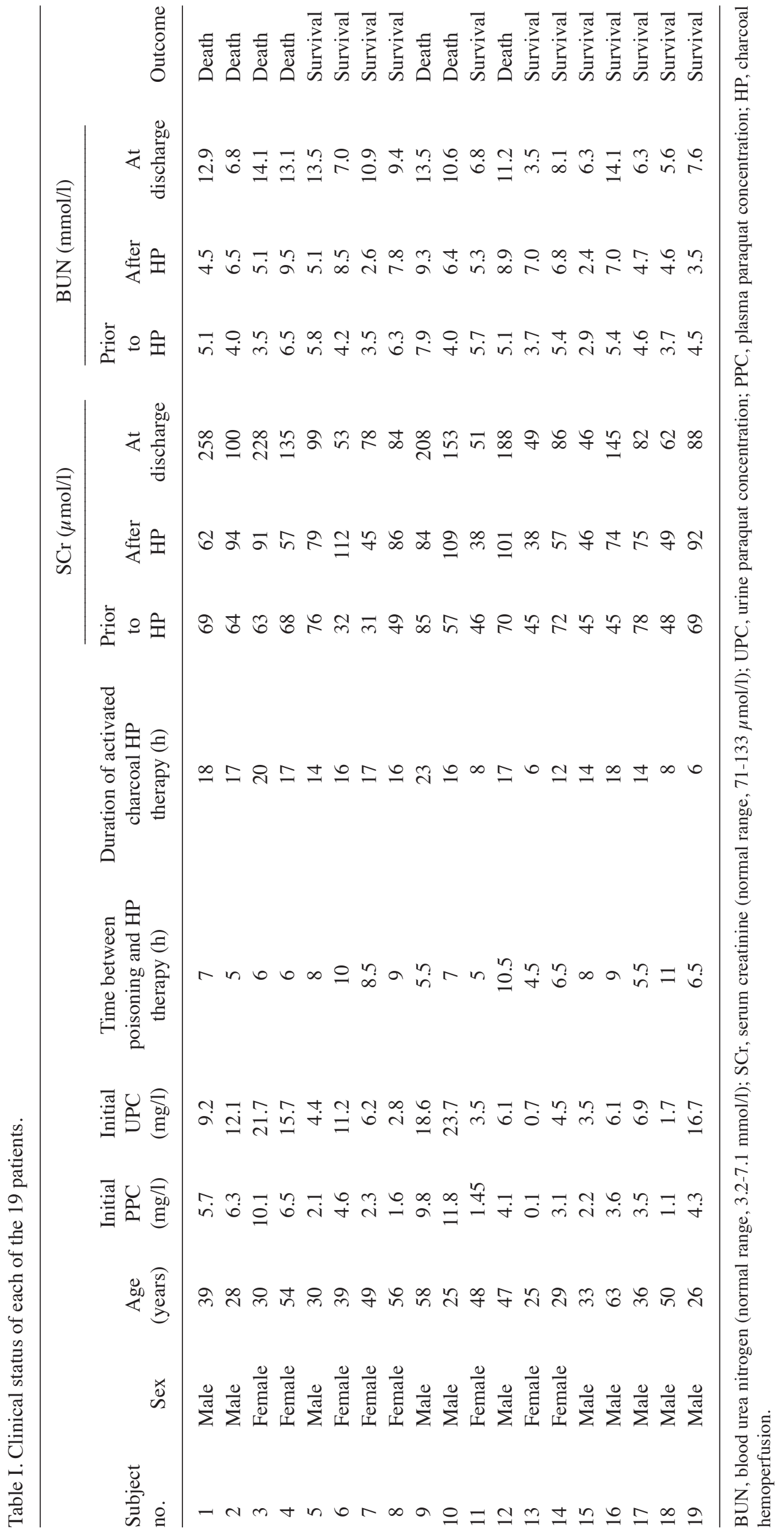




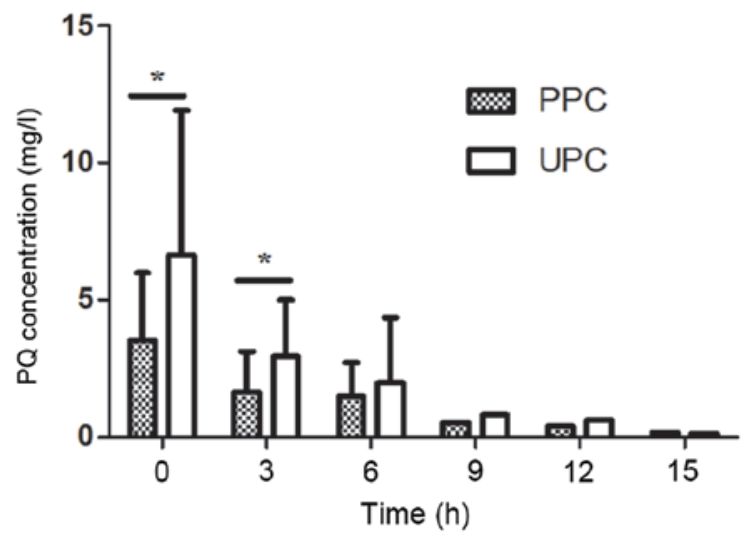

Figure 1. PPC and UPC at various time-points during HP therapy. Prior to HP therapy (0 h): PPC, $3.50 \pm 2.493 \mathrm{mg} / \mathrm{l}$; UPC, $6.66 \pm 5.25 \mathrm{mg} / \mathrm{l}(\mathrm{n}=19)-; 3 \mathrm{~h}$ after HP therapy: PPC, $1.65 \pm 1.477 \mathrm{mg} / \mathrm{l}$; UPC, $2.94 \pm 2.085 \mathrm{mg} / \mathrm{l}(\mathrm{n}=17) ; 6 \mathrm{~h}$ after HP therapy: PPC, $1.48 \pm 1.228 \mathrm{mg} / \mathrm{l} ; \mathrm{UPC}, 1.98 \pm 2.362 \mathrm{mg} / \mathrm{l}(\mathrm{n}=10) ; 9 \mathrm{~h}$ after HP therapy: PPC, $0.50 \mathrm{mg} / 1$; UPC, $0.80 \mathrm{mg} / \mathrm{l}(\mathrm{n}=3) ; 12 \mathrm{~h}$ after HP therapy: PPC, $0.40 \mathrm{mg} / \mathrm{l}$; UPC, $0.60 \mathrm{mg} / \mathrm{l}(\mathrm{n}=1) ; 15 \mathrm{~h}$ after HP therapy: PPC, $0.15 \mathrm{mg} / \mathrm{l}$ UPC, $0.1 \mathrm{mg} / 1(\mathrm{n}=1) .{ }^{*} \mathrm{P}<0.05$ as indicated. UPC, urine paraquat concentration; PPC, plasma paraquat concentration; HP, charcoal hemoperfusion.

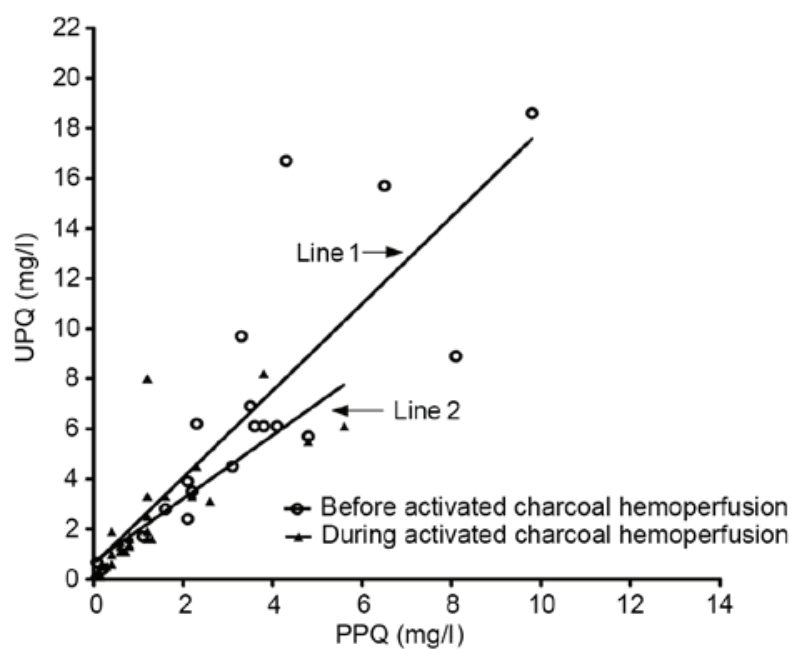

Figure 2. Line 1, correlation between PPC and UPC prior to HP therapy. Regression line, $\mathrm{Y}=0.5820+1.7348 \mathrm{X}\left(\mathrm{R}^{2}=0.678 ; \mathrm{F}=35.768 ; \mathrm{P}<0.0001\right)$; Line 2, correlation between PPQ and UPQ during HP therapy. Regression line, $\mathrm{Y}=0.6827+1.2649 \mathrm{X}\left(\mathrm{R}^{2}=0.626 ; \mathrm{F}=50.308 ; \mathrm{P}<0.0001\right)$. $\mathrm{PPC}$, plasma paraquat concentration; UPC, urine paraquat concentration; HP, charcoal hemoperfusion.

mild-to-moderate PQ-poisoning, and is ineffective for patients with severe PQ poisoning. It is likely that the potentially damaging concentration of PQ had already been attained in the kidney when HP was performed.

Finally, it should be noted that, even though renal function is normal in the early stage of PQ poisoning, and the toxin was removed effectively, this did not affect the clinical outcome in patients who had ingested a potentially lethal dose of PQ.

In conclusion, the present study suggested that the kidney toxicokinetics of PQ were only associated with the plasma PQ concentration in patients with normal renal function. Activated charcoal HP had little effect on avoiding acute kidney injury in patients with severe PQ poisoning. 


\section{Acknowledgements}

The authors are grateful to the Basic Research Project of the Logistics University of Chinese People's Armed Police Force (grant no. WHJ2015020) for financially supporting the present study.

\section{References}

1. Jones GM and Vale JA: Mechanisms of toxicity, clinical features, and management of diquat poisoning: A review. J Toxicol Clin Toxicol 38: 123-128, 2000

2. Hsu CW, Lin JL, Lin-Tan DT, Chen KH, Yen TH, Wu MS and Lin SC: Early hemoperfusion may improve survival of severely paraquat-poisoned patients. PLoS One 7: e48397, 2012.

3. Kang MS, Gil HW, Yang JO, Lee EY and Hong SY: Comparison between kidney and hemoperfusion for paraquat elimination. J Korean Med Sci 24 (Suppl): S156-S160, 2009.
4. Pavan M: Acute kidney injury following paraquat poisoning in india. Iran J Kidney Dis 7: 64-66, 2013.

5. Hawksworth GM, Bennett PN and Davies DS: Kinetics of paraquat elimination in the dog. Toxicol Appl Pharmacol 57: 139-145, 1981.

6. Hu L, Hong G, Ma J, Wang X, Lin G, Zhang X and Lu Z: Clearance rate and BP-ANN model in paraquat poisoned patients treated with hemoperfusion. Biomed Res Int 2015: 298253, 2015.

7. Pond SM, Rivory LP, Hampson EC and Roberts MS: Kinetics of toxic doses of paraquat and the effects of hemoperfusion in the dog. J Toxicol Clin Toxicol 31: 229-246, 1993.

8. Hong SY, Yang JO, Lee EY and Kim SH: Effect of haemoperfusion on plasma paraquat concentration in vitro and in vivo. Toxicol Ind Health 19: 17-23, 2003.

9. Posecion NC, Ostrea EM and Bielawski DM: Quantitative determination of paraquat in meconium by sodium borohydride-nickel chloride chemical reduction and gas chromatography/mass spectrometry (GC/MS). J Chromatogr B Analyt Technol Biomed Life Sci 862: 93-99, 2008.

10. Ikebuchi J: Evaluation of paraquat concentrations in paraquat poisoning. Arch Toxicol 60: 304-310, 1987. 